\title{
LA RECONSTRUCCIÓN DE PALEOAMBIENTES CUATERNARIOS: IDEAS, EJEMPLOS Y UNA SÍNTESIS DE LA EVOLUCIÓN DEL HOLOCENO EN EL NW DE LA PENÍNSULA IBÉRICA
}

\author{
Antonio Martínez Cortizas *
}

Abstract

\begin{abstract}
Reconstructing Quaternary palaeoenvironments: ideas, examples and a synthesis of the Holocene evolution of Northwestern Iberain Peninsula
\end{abstract}

This paper is the consequence of an invited presentation given at the Facultade de Letras do Porto during the II Jornadas do Quaternário "Quaternary, Natural and Cultural Heritage» held at Porto (Portugal). It deals with a personal view on how to reconstruct Quaternary palaeonvironments, based on the experience gained during the last litteen years collaborating with researchers of many disciplines, although 1 have to aknowledge to archaeologists and prehistoricians for triggering my interest.

Present environment can be considered as a dynamic system of emergent complexity, product of the many interactions -feedbacks, couplings, perturbations, inductions, metachronicities, ...- stablished amongst its constituent parts -basically the lithosphere, the atmosphere, the hydrosphere and the biosphere; playing humans an increasing role among biota.. So have to be considered past environments or palaeoenvironments. This complexity demands an integrated, interdisciplinary view of reasearch for a reasonable reconstruction. The object of analysis is the archive, an entity that contains a record of envirommental changes -polar and glacier ice and snow, lake and ocean sediments, peat bogs, tree rings, ...-While any property resulting from a change that can be measured and interpreted can be considered as a signal -biotic or abiotic-. Natural archives are the memory of the geosystem.

Under the influence of environmental conditions superficial formations of the lithosphere undergo processes that are responsible for their properties -physico-chemical, mineralogical, biological, etc, ...In this sense, palacoenvironmental reconstruction tries to follow the inverse intinerary: from present observed propertics to stablish the processes related to their genesis and from those we try to uncover the past environments that governed them. Nevertheless, we have to be aware of the fact that progressive and regressive pathways are both likely to have ocurred, so information was sometimes stored and sometimes deleted from the archives. In fact, the further we go back in time the less information we are able to obtain. It is also important to remind that reconstruction is always partial, as it is impossible to obtain clues of all the complexities, and even some past environments may have had conditions which are not comparable to any present environment.

With these ideas as background framework I briefly introduce here some aspects on the properties of archives and signals, propose an approach to Quaternary palaeoenvironments reconstruction, give some insights on the relationships between human activities, archaeological sites and past environments, put some examples of archives we have been using and results obtained, and end up with a synthesis of the Holocene evolution of Northwestern Spain.

Key-words: Palaeoenvironmets, reconstruction, systemic evolution, archives and signals, archacological sites, Holocene evolution, Galicia (NW Spain).

\section{INTRODUCCIÓN}

Hace algún tiempo comencé una de las conferencias del Curso de Especialización en Evaluación y Gestión del Patrimonio Cultural y Arqueología del Paisaje, celebrado en Santiago de Compostela, con la siguiente pregunta: ¿Debe ser el paleoambiente objeto de estudio como bien del patrimonio natural y cultural de un área? La intención era hacer llegar a los profesionales de la arqueología mi inquietud por la puesta en valor del paleoambiente y la necesidad de llevar a cabo estudios interdisciplinares sobre el Cuaternario como fuente para la comprensión del funcionamiento de los ecosistemas, de los medios en los que se desarrolló la actividad humana pretérita, de los procesos de cambio naturales y los inducidos por dicha actividad y como registro útil para una mejor comprensión de los futuros escenarios de cambio ambiental.
Poco tiempo después recibí una amable invitación para participar en las II Jornadas do Quaternário, bajo el lema «O Quaternário, Patrimonio Natural e Patrimonio Cultural», así que no sorprenderá mi rápida aceptación y mi agrado por esta convergencia de ideas. Este texto es, de hecho, un reflejo parcial de lo expuesto durante dichas jornadas, celebradas en la Facultade de Letras da Universidade do Porto, y patrocinadas por la $A P E Q$.

Tras casi dos décadas de investigación paleoambiental en el noroeste de la Península Ibérica, abordada con investigadores de numerosas disciplinas (Arqueología, Edafología, Geología, Geomorfología, Paleontología, Palinología, Prehistoria, etc...), he asistido a un desarrollo constante -pero a veces limitado e inconexo - del conocimiento y de la metodología empleada para la reconstrucción. Así como a intentos de crear disciplinas de sintesis multidisciplinar, de

\footnotetext{
* Grupo de Estudios Ambientales (GEA), Departamento de Edafología y Química Agrícola, Facultad de Biología, Campus Sur s/n, Universidad de Santiago de Compostela, E-15706, España. E-mail: edantxon@usc.es
} 
las cuales es un buen ejemplo la Geoarqueología - no exenta de criticas-.

Aunque a distinta escala, esto no ha sido ajeno a la evolución mundial de la investigación sobre el Cuaternario y al impacto que algunos descubrimientos han tenido en la comunidad científica, la clase política y el público en general. Un ejemplo paradigmático es la difusión que han tenido las investigaciones realizadas en los hielos polares, muy en particular la detallada reconstrucción de la evolución del clima del planeta durante los últimos 400.000 años a partir del testigo de Vostok, en la Antártida. Esta investigación ha transcendido el ámbito de las revistas especializadas.

A este panorama hemos de añadir, no obstante, algunos elementos que distorsionan una idílica imagen de progreso: el escaso conocimiento que poseemos de vastas áreas continentales y oceánicas, el fuerte desequilibrio en el desarrollo metodológico y las aproximaciones empleadas, la compleja integración de resultados, las dificultades para abordar una verdadera investigación interdisciplinar $-\mathrm{y}$ no multidisciplinar $\mathrm{y}$, también, la falta de un corpus teórico amplio en el que se base y fundamente la investigación. Este último aspecto se encuentra entre mis obsesiones particulares y se hizo todavía más patente tras asistir a la última reunión de la Commission on the Holocene (Environmental Changes During the Holocene), celebrada en marzo de 2000 en Sevilla. Mas allá de casos individuales, no se trata sólo del desconocimiento de las técnicas disponibles en la actualidad o su complicada incorporación a los proyectos en marcha por falta de presupuesto; se trata más bien de importantes desequilibrios en la filosofia de fondo que dirige la investigación. Esta visión era compartida por una parte de los asistentes a la reunión, conscientes de la necesidad de promover una síntesis.

Así pues, dentro siempre del sesgado campo de mi experiencia personal en la investigación en Ciencias de la Tierra, este artículo trata de aportar algunos elementos para el debate de fondo sobre la reconstrucción de los paleoambientes y el papel desempeñado por la actividad humana pretérita. En una primera parte pretendo introducir una serie de conceptos básicos sobre la evolución ambiental-desde una concepción sistémica-, ideas generales sobre la reconstrucción paleoambiental, los archivos y las señales ambientales, una aproximación metodológica para la reconstrucción, así como un breve comentario sobre la actividad humana en el contexto de los paleoambientes. En la segunda parte, comentaré algunos de los resultados recientes obtenidos en el noroeste peninsular utilizando archivos y señales geoquímicas, que ilustran los conceptos discutidos en la primera.

La Naturaleza escribe libros sobre su historia que nosotros debemos leer, aunque ello exige un esfuerzo de comprensión de los signos, palabras y frases escri- tas en lenguajes que, aunque naturales, no forman parte de nuestro bagaje cultural habitual. Nosotros, los investigadores del Cuaternario, hemos de plantearnos como objetivo divulgar este conocimiento para facilitar su puesta en valor y que las sociedades lo reclamen como parte de su patrimonio.

\section{Evolución AMBIENTAL: Evolución SISTÉMICA}

A escala planetaria podemos considerar la Tierra como un gran sistema, el geosistema, caracterizado por estar formado por numerosos componentes o subsistemas y poseer una dinámica compleja a distintas escalas espaciales y temporales. En lo que se refiere a procesos superficiales, los subsistemas más importantes son la litosfera, la hidrosfera, la atmósfera y la biosfera - a los que hay que añadir la influencia decisiva de la energía procedente del Sol-; aunque es susceptible de una subdivisión más pormenorizada, ad hoc, dependiendo del nivel de estudio (criosfera, edafosfera, etc...). Parece también apropiado admitir que existe una cierta invarianza de escala en los procesos que operan, en lo que podríamos definir como un funcionamiento algorítmico - en el sentido defendido por DENNETr (1999) para la evolución biológica y tal como lo define BerLINSKI (1999)-: la repetición de funciones básicas, regidas por reglas o leyes simples que, sin embargo, tienen como resultado la complejidad observada.

Bajo esta concepción, organizada como una jerarquización de aproximaciones científicas (la visión estática, de los componentes esenciales y sus propiedades en cada subsistema; la visión dinámica, de la reactividad y organización estructural, espacial y temporal de los mismos), la visión sistémica engloba conceptos tales como el de interacción, retroalimentación, interdependencia, acoplamiento, metacronicidad, perturbación o inducción (figura 1). Todos ellos se refieren a los cambios en los estados de un sistema como consecuencia de las transferencias de masa y/o energía dentro y fuera del mismo; aunque en esta breve exposición no abordaremos su definición.

La complejidad emergente de los sistemas dinámicos complejos, tal como es definida BAR-YAM (1997), es uno de los motivos por los que la investigación ha tomado un camino cada vez más reduccionista, acompañado de una creciente hiperespecialización y la pérdida de una visión mas integrada del medio ambiente. El estudio de los componentes de cada sistema tiene como objetivo el conocimiento de las propiedades y procesos básicos, a escalas que nos son abordables. Pero, finalmente, cuando el objetivo es la compresión de la compleja realidad, no queda más remedio que intentar una integración que simule lo mejor posible las interacciones entre las partes. Esto ha cristalizado en la formulación de modelos físico- 


\section{SISTEMA}

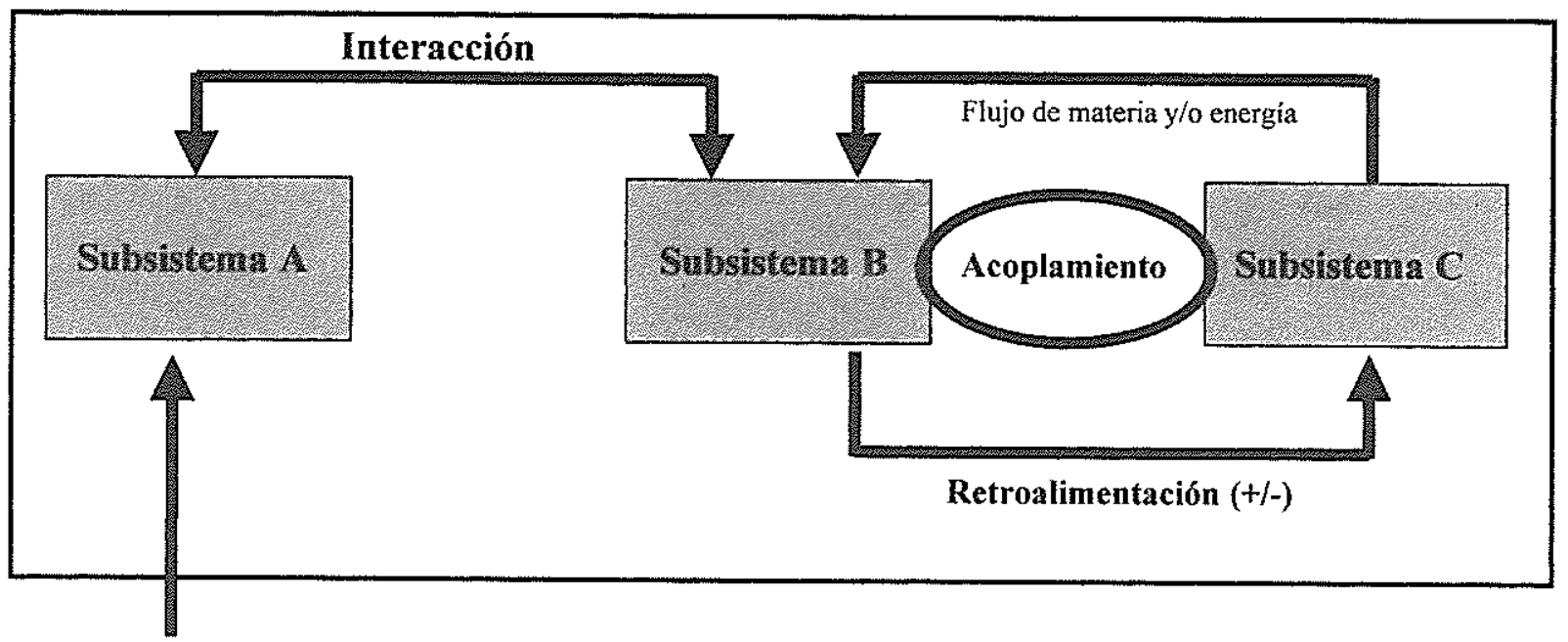

\section{PERTURBACIÓN}

Fig. I - Esq̧uema simplificado de algunos conceptos sobre la evolución de los sistemas, mencionados en el texto

matemáticos (por ejemplo, los modelos de circulación general atmosférica), hoy asistidos por ordenadores, que permiten reproducir de forma virtual el funcionamiento de los sistemas. Estos modelos ayudan a mejorar nuestra comprensión de los fenómenos y de su intrincada red de interacciones $y$, cuando se han validado con observaciones fiables, ayudan a explorar el campo especulativo de la predicción de escenarios futuros.

En mi opinión, conviene tener siempre presente que la nuestra es una investigación de experimentos que ya han tenido lugar, en un planeta - la Tierra - que es, a su vez, un experimento singular en nuestro universo conocido. El objetivo integrador debiera ser la comprensión del funcionamiento del geosistema y sus partes a diversas escalas espaciales y temporales. $Y$ es aquí donde los estudios del Cuaternario se consitituyen en un elemento básico de la ciencia, pues el pasado es también la clave del presente y del futuro. En la medida en que mejoren nuestros conocimientos y desarrollemos una base de datos potente, integrada y depurada, mejorará también la comprensión de la variabilidad intrínsica al comportamiento de los ecosistemas naturales, se desarrollarán modelos más fiables y, con ello, se podrá poner en perspectiva los cambios actuales observados y las predicciones futuras. Sin duda, esto redundará en políticas medioambientales mejores y en un enfoque menos antropocéntrico del estudio de nuestro planeta.

\section{La Reconstrucción PaLeoAmbiental}

El ambiente es el conjunto de condiciones bióticas y abióticas dominantes en un espacio dado y en un tiempo dado. Esto incluye el clima, el soporte geológico, las formas del terreno, los suelos, la fauna y la vegetación, etc..., además de sus múltiples interacciones e interdependencias, ocurridas en sincronía con la actividad humana. De una forma sencilla, el paleoambiente puede definirse como las condiciones bióticas y abióticas dominantes en un espacio y tiempo pretéritos. A lo que habrá de unirse los procesos de acoplamiento o metacronicidad entre la actividad humana y los factores ambientales, clave para entender la evolución del paisaje Holoceno, por ejemplo. Esta definición oculta, en su sencillez, el dilema de la división del continuo temporal, la dificultad de poner un límite a lo que es pasado (paleo) y lo que es presente. Esto ha llevado a algunos investigadores a defender la visión extremista de que todo es paleo a partir del momento en que ha sucedido.

La reconstrucción paleoambiental se encargaría pues del análisis de las evidencias almacenadas en archivos naturales y antrópicos, que pueden ser interpretadas con las técnicas disponibles en la actualidad. Un aspecto importante a tener en cuenta es que el registro paleoambiental es siempre fraccionario, debido en parte a que no todas las condiciones ambientales son reflejadas por los archivos, pero también a que la evolución progresiva (de aumento de la anisotropía y la información almacenada) no es la única vía evolutiva. La evolución regresiva, implicada en la pérdida de registro, es otra ruta operativa. El equilibrio dinámico entre regresión y progresión en la formación de los archivos paleoambientales y la sensibilidad que muestran algunos de ellos a las condiciones iniciales, hacen que su evolución se pueda considerar de tipo caótico determinista - tal como especulan JOHNSON ET AL. (1990) para la evolución de 
los suelos-. En cualquier caso, se ha de tener presente el carácter de palimpsesto del registro paleoambiental, ya que la información recuperable disminuye exponencialmente para los ambientes más antiguos.

En el presente, las condiciones ambientales (incluyendo la actividad humana) son las que dirigen los procesos de cambio (deposicionales y post-deposicionales) que operan en un paisaje dado, y estos procesos son responsables de las propiedades actuales que podemos determinar en las formaciones superficiales. Aplicando un sentido genético a esta descripción, la reconstrucción es el intento de recorrer el itinerario opuesto: mediante el estudio de las propiedades actuales establecer los procesos implicados en la génesis de las mismas y las condiciones ambientales dominantes (bióticas y abióticas) que debieron regular a estos últimos (figura 2). Con este fin, la aplicación del tan recurrido e importante principio del actualismo es muy útil, pero ha de advertirse que algunos paleoambientes pueden no tener un referente actual adecuado. El actualismo se aplica a los procesos y no debe ser sinónimo de presentismo - suponer que el presente y el pasado han sido iguales-.

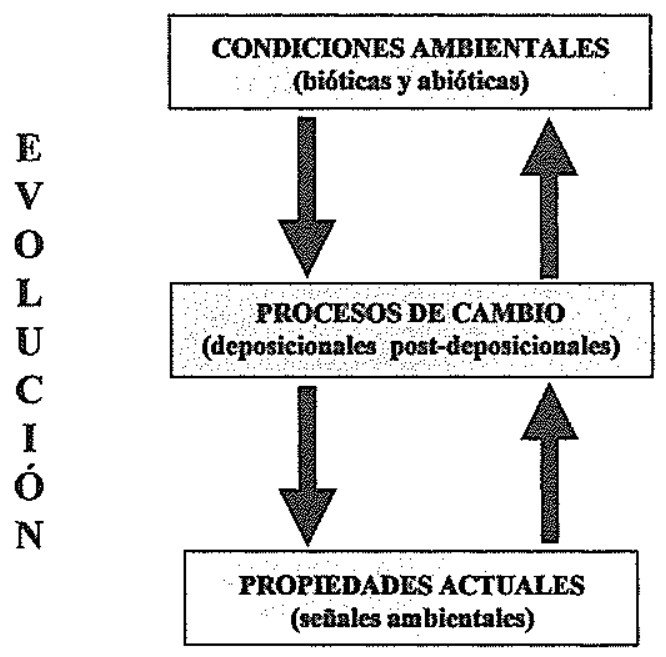

$\mathbf{R}$

$\mathbf{E}$

C

O

$\mathbf{N}$

$\mathbf{S}$

T

$\mathbf{R}$

$\mathbf{U}$

C

C

I

ó

$\mathbf{N}$

Fig.a 2-La reconstrucción paleoambiental sigue, en su esquema metodológico, el itinerario opuesto a la evolución natural de los sistemas tratando averiguar, a partir de las propiedades actuales de los archivos, los procesos implicados en su registro y las condiciones ambientales que los gobernaron.

\subsection{Archivos y señales paleoambientales}

Toda propiedad de una formación superficial, resultado de un proceso de cambio, que puede ser interpretada en términos de reconstrucción es una señal ambiental; mientras que las formaciones que almacenan las señales son los archivos ambientales. Los archivos cumplen, entonces, la función de memoria del geosistema. Por su origen podemos agruparlos en naturales (hielos árticos y de glaciares, sedimentos oceánicos y lacustres, turberas, brañas, suelos policíclicos, etc...) y antrópicos (yacimientos arqueológicos, suelos de cultivo, bancales, etc...). Por la continuidad del registro podemos clasificarlos como continuos (o cuasi-contínuos, con alta resolución para un periodo de tiempo largo) y discontinuos (que sólo han registrado uno o pocos eventos separados por hiatos temporales largos).

Por lo que se refiere a las señales, hay tres propiedades importantes (GUARDÁNS, 1991): la resolución, el margen y la extensión. La resolución es la distancia mínima con la que puede ser leída la señal; el margen, es la relación entre la intensidad del cambio producido y su registro en el archivo (relación señal/ruido); y la extensión, es la distancia temporal total del registro con señales interpretables. Las señales pueden ser de muy diverso tipo: geomorfológicas, sedimentarias, edáficas, bióticas, geoquímicas, etc..., algunas apreciables a simple vista y muchas que sólo es posible revelar tras un análisis detallado del archivo (el registro invisible).

Los archivos poseen, en general, una cierta capacidad para oponerse a los cambios internos en respuesta a las condiciones externas (amortiguación), es decir, una inercia o tendencia a conservar su estado inicial (producto de su estructura actual resultado, a su vez, de un proceso adaptativo) y a no generar un aumento de la anisotropía. Existe pues un límite mínimo, o umbral, que debe ser superado para que se produzca un cambio y se registre la señal (sensibilidad del archivo). Este umbral es determinante para fijar el tiempo de reacción del archivo, el cual ha de estar en consonancia con la periodicidad de los cambios ambientales para que éstos queden reflejados. Un archivo con un tiempo de reacción largo (alta inercia, umbral elevado) sólo registrará procesos que ocurran a intervalos temporales largos. Además, tal como he mencionado antes, en el transcurso del tiempo parte de las señales almacenadas por un archivo ambiental son susceptibles de ser eliminadas total o parcialmente por procesos post-deposicionales que tienden a reducir la anisotropía (procesos proisotrópicos), dando lugar a una pérdida de memoria.

\subsection{Los métodos para la reconstrucción paleoambiental}

La realidad ambiental, tanto actual como pretérita, es multivariante y contingente. La investigación paleoambiental debe aspirar a reconstruir la mayor cantidad de factores y parámetros posible, y obtener una integración razonable de los mismos para una buena reconstrucción. El objetivo general será el de averiguar los procesos ocurridos y las condiciones ambientales responsables de los mismos, así como esta- 
blecer la fiabilidad de las señales y los archivos analizados.

El estudio se articulará a diversas escalas que van desde lo macro a lo micro, desde las formaciones geológicas y geomorfológicas, pasando por los tipos de sedimentos y suelos, hasta el estudio sus propiedades o de los denominados registros invisibles (señales geoquímicas, fitolitos, polen, ...). Las herramientas de análisis son, a su vez, extraordinariamente numerosas.

De forma simplificada, las disciplinas a considerar se pueden agrupar en:

- Disciplinas de las Ciencias de la Tierra: consideraremos aquí a la Geomorfología, la Sedimentología, la Estratigrafia, la Geología, la Edafología, la Micromorfología y la Geoquímica, además de las técnicas informáticas aplicadas a los estudios de dimensión geográfica, como los sistemas de información geográfica.

- Disciplinas que se encargan del estudio del registro fósil o subfósil: se considerarán aquí la Palinología, la Antracología, la Paleontología, el estudio de diatomeas, el estudio de fitolitos o la Dendrocronología y la Dendroecología.

- Disciplinas que se encargan del estudio de los restos materiales de la cultura: no se puede desligar la evolución ambiental pretérita de la de los grupos humanos que han ocupado un determinado espacio, por ello la Arqueología y la Prehistoria son disciplinas que deben participar de forma activa en la recons trucción paleoambiental.

Desde el punto de vista metodológico, el estudio puede articularse en cuatro fases (figura 3). De modo operativo, se debe comenzar por una primera fase de gabinete en la cual se recopilará toda la información disponible sobre el área (cartografia, datos climáticos, geología, hidrografía, estudios previos, etc.), que ha de ayudar a la comprensión de las peculiaridades actuales del medio objeto de estudio. Esta fase ha de servir, asimismo, para fijar las primeras estrategias a seguir en la planificación del trabajo. En una segunda fase se procederá a la realización de los trabajos de campo. Una etapa preliminar debe encaminarse a la caracterización geomorfológica, sedimentológica, estratigráfica y edafológica, para identificar las unidades del terreno y elucidar los procesos de formación que han operado a escala de paisaje. La sectorización, necesariamente con base cartográfica georeferenciada-y con preferencia en soporte digital tipo SIG-, es imprescindible para la toma de decisiones sobre los archivos potenciales y las áreas que han de ser objeto prioritario de investigación en profundidad. Una vez que se han seleccionado las áreas potenciales de actuación y los tipos de archivos que éstas ofrecen para la reconstrucción paleoambiental, es necesario redefinir las disciplinas implicadas en el estudio, asi como las metodologías a emplear en cada caso (tanto en el proceso de toma de muestras como en la analítica de laboratorio). Conviene destacar, desde un principio, que estos dos aspectos están supeditados a los objetivos de la reconstrucción, ya que son estos últimos los que ayudarán a decidir entre el amplísimo espectro de técnicas y disciplinas que pueden aportar información sobre los ambientes pretéritos.

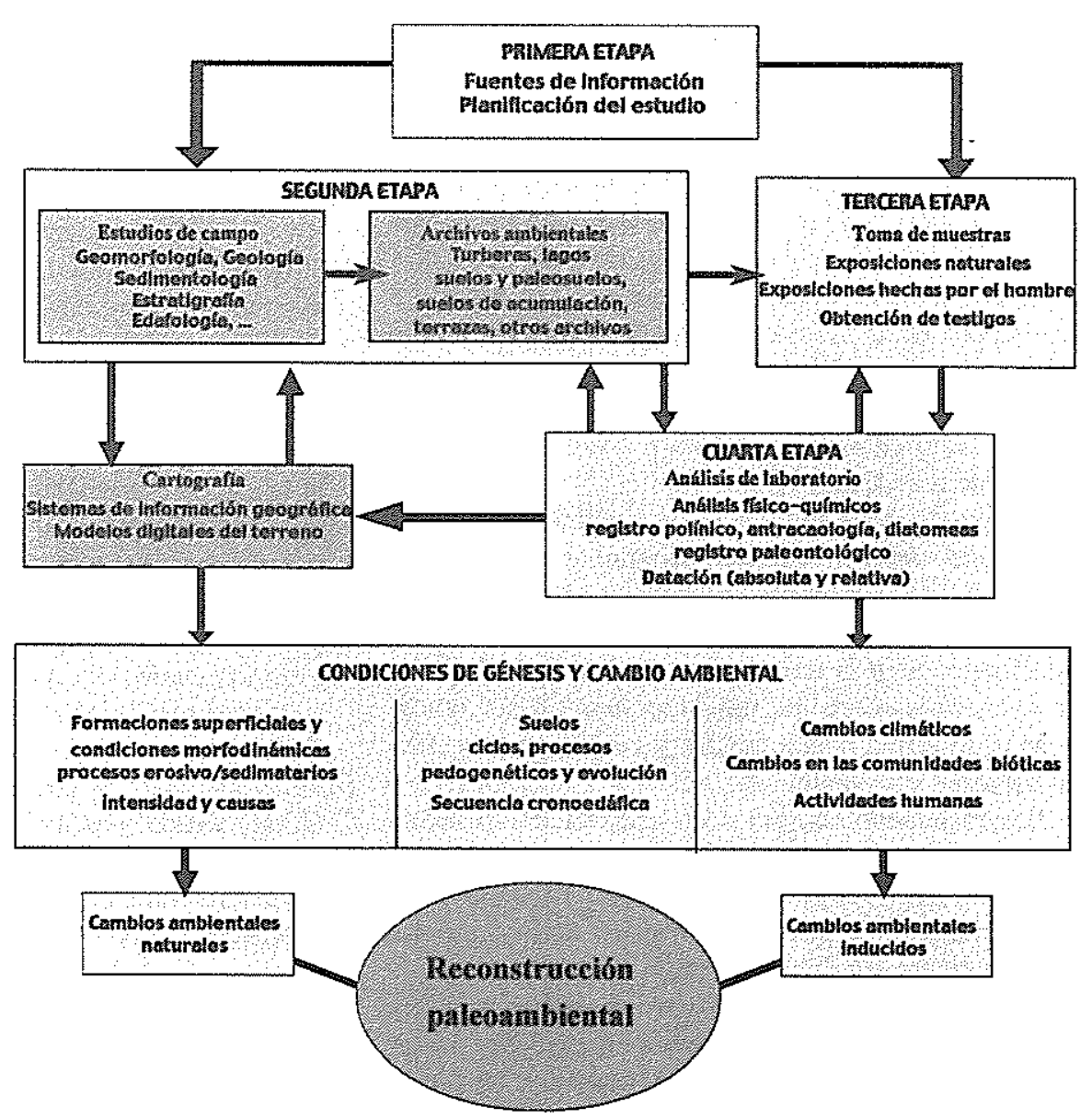

Fig. 3-Esquema de las fases propuestas para el desarrollo de los estudios de reconstrucción paleoambiental sobre el Cuaternario. 
En una tercera fase se ha de proceder a la toma de muestras de los archivos elegidos. Esta ha de contemplar dos aspectos básicos: por un lado, los métodos de sondeo y por otro el intervalo de muestreo en cada depósito o archivo paleoambiental. Cuando existen exposiciones de las formaciones sedimentarias $y$ suelos, la toma de muestra puede realizarse sin gran esfuerzo; $\sin$ embargo, algunas unidades con un elevado potencial de información paleoambiental - como las turberas, los sedimentos lacustres, las brañas o los suelos de bancales - habitualmente no ofrecen exposiciones adecuadas, por lo que ha de procederse a la toma de testigos mediante sondeo o apertura de zanjas. El primero de estos métodos provoca un impacto menor sobre las formaciones de estudio, pero tiene las limitaciones de la reducida cantidad de muestra que se obtiene y la incertidumbre introducida por las posibles variaciones laterales de las formaciones sedimentarias que contenga el depósito, que pueden sesgar notablemente la interpretación de las secuencias cronoedáfica, estratigráfica, etc - sólo solucionable con un sondeo múltiple-. El segundo obvia estos inconvenientes, pero no siempre es factible aplicarlo dado el coste que implica y el conflicto que puede suponer con la propiedad de las áreas en las que se desarrolla el trabajo.

En todos los casos, el intervalo de muestreo ha de ser acorde con la resolución temporal que ofrezca el archivo que se analiza y con los objetivos cronológicos propuestos en la reconstrucción. Para las unidades que sean homogéneas - en cuanto a niveles estratigráficos y morfología de los suelos-, un muestreo sistemático es la mejor de las pautas a seguir. Cuando estén presentes unidades heterogéneas, la toma de muestras ha de tener en cuenta la variabilidad de cada nivel o ciclo edáfico, pudiendo optarse por sistemas mixtos de muestreo.

La cuarta fase atañe a los procedimientos analiticos, que también pueden programarse de forma secuencial, de manera que los datos que se vayan obteniendo ayuden a comprender los procesos implicados y retroalimenten la tercera fase, permitiendo la toma de decisiones sobre qué unidades han de ser muestreadas de forma más intensiva y cuales no necesitan un esfuerzo suplementario.

Los resultados obtenidos en estas cuatro fases se encaminarán hacia la resolución de: 1) la génesis de las formaciones superficiales del área, las condiciones morfodinámicas implicadas y el papel de la actividad humana en los cambios superficiales; 2) el establecimiento de los procesos erosivo/sedimentarios que han tenido lugar y sus posible causas; 3 ) la caracterización de las principales rutas edafogenéticas, los ciclos de suelos y la secuencia cronoedáfica local y su relación con la regional; 4) los cambios en las comunidades bióticas y su cronología; 5) los cambios climáticos;
6) los usos del territorio por parte de los grupos humanos, así como sus repercusiones a escala de paisaje. En conjunto, todos estos objetivos van dirigidos hacia la discriminación de los procesos de cambio en épocas pretéritas, su origen natural o antrópico y las interacciones y acoplamientos entre cambios ambientales y cambios culturales.

\subsection{Actividad humana pretérita, yacimientos arqueológicos y entorno}

Tras un largo periodo de colaboración con investigadores de los campos de la Arqueología y la Prehistoria, en lo que se refiere a la reconstrucción de los ambientes pasados en los que se desarrolló la actividad humana, se ha ido urdiendo una discusión de base sobre cómo enfocar las relaciones hombremedio (Llana ET AL., 1992 e 1993; Martinez CorTizAS ET AL., 1993, 1994; MARTínez CoRTIZAS E MoARES Dominguez, 1995, 1996a,b; Martínez Cortizas E LlANA RodRíguez, 1996, 1997). Sin pretender exponer aquí una visión única, mencionaré tan sólo brevemente algunas ideas de cómo percibo esta temática en la actualidad. Mi intención es contribuir a la reflexión, puesto que la discusión ha sido a veces acalorada; muy en particular por la dificultad que entraña para los historiadores y prehistoriadores aceptar las influencias externas a la cultura como fuerzas directoras, y a la facilidad con la que otros científicos - en general de las Ciencias de la Tierra - asumen, a veces de manera simplista, el paradigma del medio como motor último del cambio cultural.

El yacimiento arqueológico ha venido siendo considerado como el objetivo central de la investigación prehistórica. Sin embargo, dado que la actividad humana raramente se circunscribe de forma exclusiva al espacio representado hoy por el yacimiento, en la investigación paleoambiental la combinación yacimiento-entorno potencialmente puede ofrecer una visión más aproximada a la compleja relación de los grupos humanos con los medios que estos habitaron y de los cuales obtuvieron sus recursos. Las aproximaciones son diversas, si bien en el contexto desarrollado hasta aquí y basándonos en los resultados obtenidos por nuestro grupo de investigación durante la última década, nos inclinamos a optar por un modelo que tome al yacimiento como un sumidero neto de recursos del medio y a su entorno como la fuente que aporta dichos recursos (bióticos y abióticos), necesarios para el mantenimiento de la cultura (con distintos radios de acción) (figura 4 ). De esta forma es posible contrastar qué recursos han sido el objeto de los grupos humanos (representados en el yacimiento), cuáles han sido preferentes (suprarepresentados) y cuáles no (infrarepresentados o ausentes), así como el impacto que la utilización de dichos recursos ha tenido 


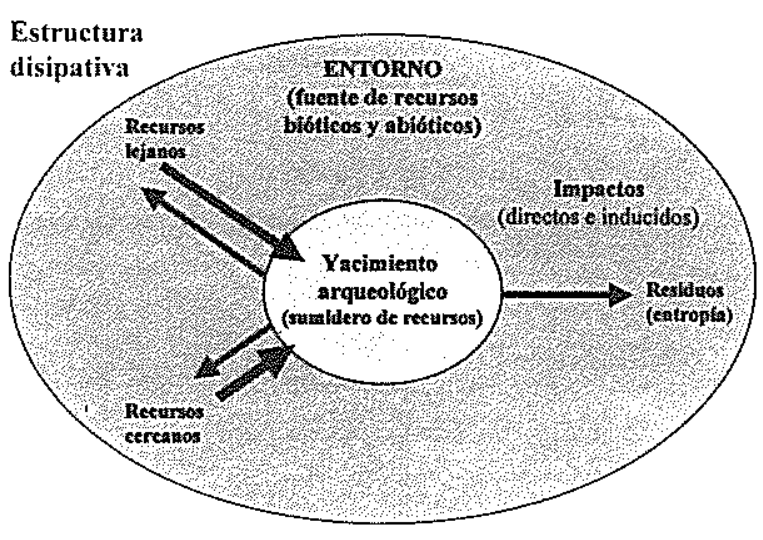

Fig. 4-Una aproximación termodinámica a los yacimientos arqueológicos y la actividad humana pretérita. Cada grupo humano que ocupa un espacio en un tiempo dado puede ser considerado como una estructura disipativa, que obtiene recursos del medio transfiriendo a al mismo la entropia resultante.

en el medio (análisis del territorio, estructuración de los espacios cercanos y lejanos, fuentes de aprovisionamiento, etc...).

La obtención y manejo de los recursos genera impactos directos, consecuencia inmediata de la actividad (deforestación, por ejemplo), e inducidos, desencadenados de forma colateral por dicha actividad (erosión de suelos, por ejemplo). Pero también produce residuos, productos secundarios no reutilizables, hacia los cuales el medio muestra una capacidad limitada de amortiguación. Impactos y residuos son una medida de la entropía generada por la actividad humana. Desde esta óptica termodinámica, la actividad de un grupo humano puede compararse a una estructura disipativa, que mantiene su funcionamiento y organización por medio del consumo de recursos del entorno y transfiere al mismo la entropía resultante. Esto permite crear modelos especulativos según los cuales el medio posee una carga crítica (discutida brevemente en MARTINEZ CORTIZAS Y LLANA, 1996), que una vez superada provoca que su capacidad para soportar la actividad humana disminuya bruscamente hasta alcanzar una entropía máxima, haciéndose insostenible el estado precendente de relación hombre-medio. Esto sugiere que podría haber un delicado equilibrio entre la extracción de recursos y la generación de impactos y residuos, que pondría un límite a la capacidad del medio para soportar una cultura dada en un tiempo dado y en un medio con unas condiciones ambientales dadas.

Además, es importante tener en cuenta que la carga crítica del medio varía con las condiciones ambientales, de tal manera que ante condiciones cambiantes (un cambio climático por ejemplo) es razonable suponer que el impacto de las actividades humanas también varíe, aunque éstas no cambien de manera sustancial. Lo más probable, no obstante, es que la actividad humana se acople a las nuevas condiciones y modifique sus relaciones con el medio, adaptándose al cambio (metacronicidad), siempre que sus recursos culturales - en sentido amplio - se lo permitan.

\subsection{Algunos ejemplos de archivos y señales}

En los últimos años, nuestro grupo de investigación (Grupo de Estudios Ambientales, GEA) se ha interesado por la aplicación de las señales geoquímicas a los estudios de reconstrucción del Cuaternario. Las bases teóricas son las descritas hasta aquí, por lo que nuestro trabajo se fundamenta en que algunas de las propiedades de las formaciones superficiales de la corteza terrestre (archivos ambientales) muestran una relación mas o menos estricta con procesos que son desencadenados por las condiciones ambientales. Las variaciones de estas propiedades, o señales paleoambientales, cumplen además el requisito de no sufrir una modificación post-deposicional importante, de tal forma que la relación señal/ruido es elevada a escalas temporales de miles de años. En otros casos, aún habiendo una modificación post-deposicional ésta puede ser modelada y sus efectos corregidos para la obtención de la señal original.

Los trabajos realizados dentro de esta línea de investigación han ahondado en los siguientes aspectos:

Estudio del cambio climático: los flujos de algunos elementos desde la litosfera e hidrosfera hacia la atmósfera y desde ésta de nuevo hacia la litosfera, están controlados por condiciones ambientales de temperatura y humedad (MARTiNEZ CORTIZAS ET AL., $1999 \mathrm{~b}, 2000 \mathrm{~b})$. Un caso paradigmático es el del mercurio $(\mathrm{Hg})$, el cual es un elemento volátil a temperatura ambiente. Estudios llevados a cabo en turberas de Galicia, han demostrado que la acumulación y estabilidad térmica de este elemento están fuertemente condicionadas por la temperatura y la humedad y que su estudio permite la reconstrucción detallada de las variaciones termopluviométricas .

Igualmente, las variaciones en las relaciones entre elementos de origen litogénico $(\mathrm{Ti}, \mathrm{Zr}, \mathrm{Sc}, \mathrm{Rb}$. $\mathrm{Sr}$, etc...) acumulados en las turberas ombrotróficas, han demostrado estar controladas por el transporte atmosférico, consituyendo un excelente estimador de la intensidad del viento.

Estudio de los procesos de erosión inducida: tres son los tipos de archivos que hemos utilizado, suelos policícliclos, suelos de acumulación (ránkeres) y turberas (Martínez CoRTIZAS, 1996; MarTínez CorTIZAS ET AL., 1993a,b,c, 1999a, 2000a,c; FrANCO MASIDE ET AL., 2000). En los primeros casos, los factores de enriquecimiento de elementos traza han sido empleados con éxito para ubicar paleosuperficies enterradas, que junto con dataciones radiocarbónicas de la materia orgánica coloidal del suelo y de carbones concentrados en el perfil, han aportado información sobre la cronología e intensidad de los episodios 
erosivos. En consonancia con ello, la transferencia de elementos litogénicos desde los suelos hacia la atmósfera, por erosión eólica, y desde la atmósfera hacia las turberas ombrotróficas, también ha servido para llevar a cabo una comprobación alternativa de la cronología e intensidad de la erosión. Ambas aproximaciones han ofrecido una visión coherente para el Holoceno, que también es apoyada por los estudios arqueológicos y prehistóricos.

Contaminación atmosférica: las variaciones atmosféricas de los flujos y composición isotópica de elementos contaminantes $(\mathrm{Pb}, \mathrm{Cd}, \mathrm{Zn}, \mathrm{Hg}, \mathrm{Cu}, \ldots)$, registrada por la deposición de los mismos en turberas ombrotróficas y sedimentos lacustres del noroeste peninsular, se han podido relacionar con fases históricas y prehistóricas de la minería y metalurgia de estos elementos (Martínez Cortizas eT al., 1997a,b, 1999b, 2001; Pontevedra POMBal et AL., 2000) (figura 5).

\section{Evolución del Holonceo en Galicia: UNA SINTESIS}

La integración de los resultados obtenidos en el marco de las investigaciones que acabamos de resumir, ha producido la sintesis más reciente de que disponemos para la evolución holocena de Galicia (figura 6). Esta revela que el noroeste de la Peninsula Ibérica ha estado sujeto a cambios ambientales importantes durante el Holoceno. Cambios que han sido el resultado de procesos naturales (cambios climáticos, por ejemplo) o bien han sido inducidos por las actividades humanas. Las investigaciones geoarqueológi-

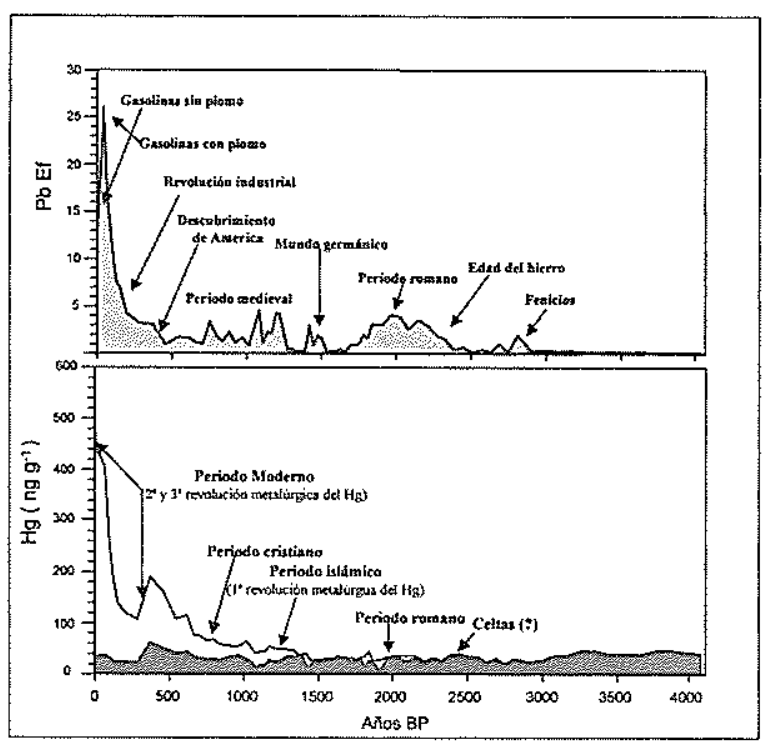

Fig. 5 - Evolución de la contaninación atmosférica por $\mathrm{Pb}$ y $\mathrm{Hg}$ en el noroeste peninsular, como resultado de las actividades mineras y metalúrgicas prehistóricas e históricas. En la gráfica del $\mathrm{Hg}$, el área sombreada en color mas oscuro representa las variaciones naturales de fondo y el área clara la contribución de origen humano.

cas y paleoambientales realizadas hasta el momento sugieren además que, en buena medida, la actividad humana ha estado en metacronicidad con los cambios de origen natural. Esto es, el desarrollo de las actividades se ha acoplado a las condiciones ambientales y la intensidad de los procesos de inducción antrópica ha dependido, a su vez, de la sensibilidad del medio en cada momento de su estado evolutivo; lo que hemos

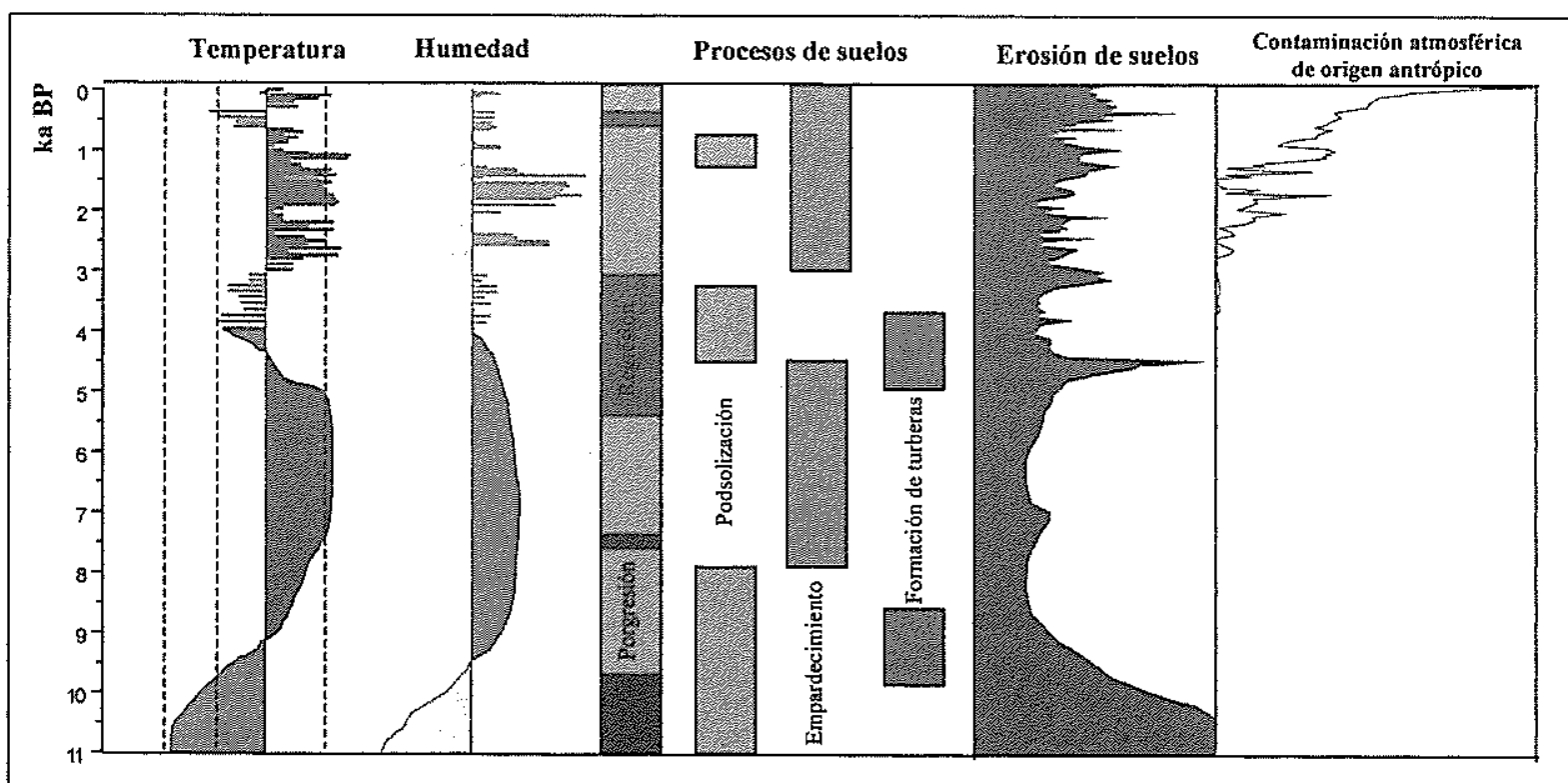

Fig. 6-Síntesis de la evolución holoceno del noroeste de la Península Ibérica (evolución climática, rutas y procesos dominantes en la evolución de suelos, intensidad de los procesos erosivos y contaminación atmosférica por metales pesados). 
denominado como carga crítica del medio (MARTíNEZ CORTIZAS Y LLANA, 1996). Es una historia dominada por los procesos de erosión y sedimentación, cambios en las formaciones superficiales y en las rutas edafogenéticas dominantes, a diversas escalas espaciales y temporales, en la cual el peso de los procesos de inducción antrópica ha seguido una progresión exponencial.

El final del Pleistoceno cursa en el NW penisular con la última gran pulsación fría reconocida. Esta pulsación está ubicada cronológicamente entre el 11.500 BP y el 9.500-10.000 BP, como atestiguan las unidades geomorfológicas, sedimentarias y edáficas pertencientes a este tramo temporal. Por ejemplo, en la Sierra del Xistral el depóstio de Río Boo contiene un paleosuelo datado en el 11.500 BP que es cortado de forma abrupta y discordante por un head de origen periglaciar que refiere un cambio a condiciones más frías y secas; mientras el inicio de acumulación de turba, tal como reflejan las dataciones basales de algunas turberas de cumbre de la misma sierra, se sitúa en torno al 9.500-9.800 BP y éstas turberas descansan, en su mayoría, sobre campos y laderas de bloques. En muchos ambientes del NW de la península son excepcionales los suelos de edad preholocena, por lo que el Dryas final ha de ser considerado como una fase relevante, desde el punto de vista ambiental, ya que en ella se generaron nuevas superficies y depósitos que sirvieron de materiales de partida para los procesos edafogenéticos que operaron durante el Holoceno.

La mejoría climática que supuso el inicio del actual interglaciar no sólo se expresó en la formación de turberas de cumbre, si no que la acumulación de materia orgánica (melanización) ligada a la progresión de la cobertura vegetal, permitió la formación de horizontes A y la puesta en marcha de la edafogénesis de las nuevas superficies. En los suelos cumúlicos que hemos estudiado hasta el momento y con independencia de su ubicación geográfica, las primeras fases de melanización ocurrieron al menos alrededor del 8.000 BP. Estos suelos han pervivido tan sólo en posiciones del paisaje de baja energía, propensas a la sedimentación. Además de la melanización, en las áreas de montaña la podsolización parece haber sido bastante intensa en esta primera etapa (MOARES DOMiNGUEZ ET AL., 1996), todavía bajo unas condiciones climáticas frías. En algunos suelos, la intensidad de este proceso dio lugar a la formación de costras ferro-húmicas, las cuales desempeñarán más tarde un papel decisivo en la infiltración del agua, en los procesos físico-químicos de los horizontes subsuperficiales e incluso en la erosión de los ciclos edafogenéticos más antiguos.

En áreas con una elevada densidad de yacimientos arqueológicos pertenecientes al Epipaleolítico, se han encontrado indicios de los que tal vez sean los primeros procesos erosivos mediados por la actividad humana (Martinez Cortizas y MoAres Domínguez, 1995). Estos cambios parecen haber sido muy localizados, circunscritos al entorno inmediato de los yacimientos arqueológicos y como resultado del uso del fuego.

Durante el óptimo Holoceno, la progresión de la edafización implica la incorporación del empardecimiento y la iluviación (en suelos desarrollados sobre materiales ricos en arcilla) a los procesos edafogenéticos dominantes. Sin embargo, a partir del 6.000-5.500 BP la erosión de los suelos comienza a generalizarse en las laderas. Los suelos ubicados en posiciones de replano o en áreas de recepción, son enterrados por matreriales ya edafizados, que poseen propiedades de horizontes $\mathrm{A}$, lo que invierte la tendencia progresiva hacia una diferenciación de los perfiles, encaminándose hacia rutas proisotrópicas regresivas en las cuales el perfil del suelo aumenta en espesor mediante una acreción superficial no asimilativa, pero no sufre diferenciación morfológica (Martinez CorTIZas, 1996). Estos espisodios parecen ser el resultado de la actividad humana, pues son sincrónicos con una disminución de la cobertura arbórea (RAMIL REGO, 1993) $y$, en algunos casos, han dejado líneas de carbones como claro reflejo de la aplicación del fuego. Por ejemplo, en el depósito de Mougás uno de los paleosuelos está enterrado por un material de ladera, hacia cuya base se encontró una línea de carbones con una edad de 5.530 años BP (Costa CASAIS ET AL., 1996); en el ranker atlántico de Currobedo (CBD) el enterramiento del paleosuelo ocurre hacia el $6.050 \mathrm{BP}$ (Martinez Cortizas et Al., 1999a) y en el de Coto Fenteira (CTF) hacia el 5.630 BP (FrAnCO MASIDE ET AL., 2000; Martínez Cortizas E'T AL., 2000a); mientras que en la cuenca de Valadouro se encontraron árboles enterrados bajo 1-2 $\mathrm{m}$ de sedimento, con una edad de 5.640 BP.

Al inicio de este periodo, la intensidad de los procesos erosivos es variable, pues mientras que en ciertos sectores los datos geoquímicos sugieren una baja o moderada intensidad, en otros los rasgos sedimentarios y geomorfológicos indican una intensidad fuerte. Lo que es común a todas las áreas estudiadas es que, a partir de este momento, las laderas pierden su equilibrio natural y sufren episodios reiterados de inestabilidad, llegando la erosión a exhumar las saprolitas o incluso los sustratos rocosos. En zonas bajas o de replano la sedimentación da lugar a una colmatación que crea nuevas superficies que, debido al aumento del flujo de agua por escorrentía asociado a la pérdida de suelo y vegetación en las laderas, en algunos sectores se transforman en suelos hidromorfos e incluso acaban por aturberarse (turberas minerotróficas y brañas). Estas áreas de relieve suave o llano son susceptibles de ser aprovechadas para actividades humanas.

Los estudios llevados a cabo en yacimientos arqueológicos ponen de manifiesto un cambio sustancial en 
las relaciones de los grupos humanos con el paisaje (Martinez Cortizas y Moares Dominguez, 1995). $\mathrm{El}$ análisis de los rankeres atlánticos de Currobedo (CBD) y Coto Fenteira (CTF), apoya esta interpretación: así, en CTF hemos encontrado dos líneas de carbones datadas en el 4.160 y el $3.300 \mathrm{BP}$, y en CBD no hay indicios de estabilidad hasta el cambio de era. En Arcucelos (Verín, Ourense) se encontró un paleosuelo que fue datado en el $3.340 \mathrm{BP}$, y que aparece enterrado por un depósito de arenas de a $6.7 \mathrm{~m}$ de espesor. Igualmente, en un estudio en curso en el área de Saa (Camposancos, Pontevedra) se han encontrado rellenos de valle que marcan el inicio de la formación de bancales en la zona, con una edad de 3.100 BP.

Cabe señalar, no obstante, que esta actividad humana tiene lugar durante un episodio de degradación climática, conocido con el nombre de Neoglaciación, para el cual se estima que las temperaturas en el noroeste ibérico pudieron haber sido de $1^{\circ}$ a $2^{\circ} \mathrm{C}$ inferiores a las actuales (MARTinez CortizAS ET AL. 1999b). Por ello, actividad humana y cambio climático pueden haber sumado sus efectos para producir una degradación del medio sin precendente pretérito (disminución de la carga crítica del medio y aumento de la presión antrópica). Durante este periodo se detecta un nuevo avance de la podsolización, en consonancia con las condiciones frías (MOARES DOMinguez ET. AL., 1996), si bien algunos autores apuntan que las modificaciones antropogénicas de la cobertura vegetal, favoreciendo el predominio de los brezales, habría colaborado en la reactivación de este proceso edafogenético (GUILLET, 1971).

Como ya se ha mencionado, no es hasta el cambio de era en que las laderas vuelven a mostrar sintomas de estabilidad, aunque no de manera permanente. El patrón evolutivo del paisaje se diversifica de forma sustancial, perviviendo incluso hasta hoy. Por ejemplo, en el área de Sar (Santiago de Compostela, A Coruña), hemos datado una generación de bancales en torno al 1.900 BP. El último episodio geomórfico relevante parece haber estado relacionado con la Pequeña Edad del Hielo (siglos XV a XVIII), habiendo sido detectado en numerosas áreas de Galicia, desde la costa hasta la montaña interior.

Por lo que atañe a la contaminación atmosférica producto de las actividades mineras y metalúrgicas, los registros indican que ésta se remonta a hace al menos unos 2.800 años BP. A partir de esta fecha los flujos naturales de $\mathrm{Pb}$ comienzan a aumentar de forma continua, hasta alcanzar un máximo pre-industrial en época romana (MARTINEZ CORTIZAS ET AL., 1997a y b, 2001). Mientras que para el $\mathrm{Hg}$ los flujos de origen antropogénico comienzan hacia el 2.400 $2.500 \mathrm{BP}$, y para el $\mathrm{Zn}$ no es hasta el $1.000 \mathrm{BP}$ que se detectan los primeros procesos de contaminación. La contaminación atmosférica parece haber seguido fielmente los abatares de la evolución histórica de la Península Ibérica, mostrando que hace unos 200-300 años - en paralelo a la revolución metalúrgica e industrial - los flujos antropogénicos de algunos elementos contaminantes sufrieron un aumento sin precendete histórico en su intensidad. Así, por ejemplo, a partir de estudios de acumulación de metales pesados en las turberas ombrotróficas (Martínez Cortizas et al., $2000 \mathrm{c}$ ) se ha establecido que de la acumulación total ocurrida en 4.000 años, en los últimos 300 se ha depositado un $23 \%$ del $\mathrm{Cu}$, un $26 \%$ del $\mathrm{Cr}$, un $45 \%$ del $\mathrm{As}$, un $55 \%$ del $\mathrm{Zn}$ y del $\mathrm{Pb}$ y hasta un $65 \%$ del $\mathrm{Mn}$.

\section{BiBLIOGRAFiA}

BAR-YAM. Y. (1997)-Dynamics of complex systems, Addison Wesley, Reading, Massachusets, $848 \mathrm{p}$.

BIERLINSKI, D. (1999) - The advent of the algorithm: the idea that rules the world, Harcourt, New York, 345p.

Costa Casals, M.; Moares Domingumz, C. E Martinez Cortzas, A. (1996) «Caracterización físico-química do depósito de Mougás (Pontevedra): implicacións morfoxenéticas", in PÉrEz Al.Blakti, A; Martini, P; ChIsworT13, C. E Martienz Cortizas, A. (eds.), Dinámica y Evoltución de Medios Cuaternarios, Xunta de Galicia, Santiago, p. 431-440.

DinneT, D. (1999)-La peligrosa idea de Darwin, Glaxia Guttenberg-Círculo de Lectores, Madrid, 926p.

Franco Maside, S.; Martinizz Cortizas, A. e Garcia-Rodisa, E. (2000) - «El mercurio como indicador de la evolución de los suelos y del paisaje durante el Holoceno en Galicia (NW de España)», in Díz bl: Otomo, F.; Faust, D. : Porras, A.l. (eds.), Environmental Changes During the Holocene, Commission on the Holocene, Universidad de Sevilla, Katholische Universität Eichstätt, AEQA, Sevilla, p. 139-142.

GUARDANS, R. (1991) - Los registros naturales en la investigación paleobiológica y paleoclimática. Algunas aplicaciones y métodos, CIEMAT/PRYMA/UCRE 15-91, 67p.

Gultel:T, B. 1987. «L'age des podzols», in Righi, D. e Chauvel, A. (eds.) Podzols et Podzolitation. Comptes Rendus de la Table Ronde International. INRA. pp: I3]-[44.

JOHNSON, D.L.; KILl.ER, E.A. E Rockwel.L, T.K. (1990)«Dynamic pedogenesis: new views on some key soil concepts and a model for interpreting Quaternary soils", Quaternary Research, 33, p. 306-319.

Llana Rodriguez, C.; Martintz Corttzas, A. E Ramil. Rego, P. (1992) - «Algunas consideraciones acerca de la estratigrafia y del marco temporal para los yacimientos al aire libre de] Pleolítico final-Epipaleolítoc de Galicia», Zephyrus, 44m45, p. $155-166$.

Llana Rodriguez, C; Martinez Cortizas, A. iz Ramil. Rego, P. (1993) - «Contribución al conocimiento de los procesos post-deposicionales en yacimietnos al aire libre con secuencia edáfica de Galician, Arqueologia Espacial, 16-17, p. 95-104.

Martinez CorTizas, A. (1996) - «Evolución de los suelos durante el Cuaternario reciente en las Sierras Septentrionales de Galicia (NW de España)", PÉrlz Alierti, A. E MARtine $\angle$ Cortizas, A (eds.), Avances en la Reconstrucción Paleoambioental de las äreas de Monfaña Lucenses, Diputación Provincial de Lugo, Lugo, p. 137-162.

Martinez Cortizas, A. E Llana Robrigulez, C. (1996)- «Una aproximación interdisciplinar a la contextualización espaciotemporal de los yacimientos al aire libre en Galicia», Almadán, 5, p. 23-36.

Martinez Cortizas, A. E Llana Rodriguez, C. (1997) - «Conceptos estratigráficos y edáficos en contextos tumulares», in Rodríguez CAsal, A. (ed.), O Neolitico Atlántico er as Orixes do Megalitismo, Consello Da Cultural Galega, Universidad de Santiago de Compostela e Unión Internacional das Ciencias Prehistóricas e Protohistóricas, Santiago, p. 73-91. 
Martinez Cortizas, A. e Monres Dominguez, C. (1995) - Edafologia y Arqueologia: estudio de yacimeintos arqueológicos al aire libre en Galicias, Xunta de Galicia, Santiago, 190p.

martinez Cortizas, A. e Moares Dominguez, C. (1996a)«Estra-tigrafias de yacimientos arqueológicos gallegos: procesos formativos y postdeposicionales», in FÁBREGAS VALCARCE, R. (ed.), Os Primeiros Poboadores de Galicia: O Paleolitico, p. 193-214.

Martinez Cortizas, A. e Moares Dominguez, C. (1996b) - «Significado paleoambiental de las estratigrafias de monumentos tumulares de la meseta superior ibérica», in PÉrEz Al.3ERTI, A; Martinl, P; Chesworth, C. e Martienz Cortizas, A. (eds.), Dinámica y Evolución de Medios Cuaternarios, Xunta de Galicia, Santiago, p. 81-95..

Martinez Cortizas, a.; fábregas Valcarce, R. e franco MASIDE, S. (2000) - «Evolución del paisaje y actividad humana en el área de Monte Penide (Redondela, Pontevedra): una aproximación metodológican, Trabajos de Prehistoria, 57, p. 173-184.

Martiniz Cortizas, A.; Garcia-Romisa, E.; Pontevedora Pombal, X. E NÓvoA MuÑoz, J.C. (2000)-«Registros geoquimicos del cambio climático holoceno: el mercurio en las turberas ombrotróficas (NW de España)», in DiAZ DiL OLoMo, F.; FAUST, D. E PORRAS, A.I. (eds.), Environmental Changes During the Holocene, Commission on the Holocene, Universidad de Sevilla, Katholische Universität Eichstätt, AEQA, Sevilla, p. 87-89.

Martiniz Cortizas, A.; Garcia-Rodeja, E.; Pontevedra Pombat. X.; Nóvon MuÑoz, J.C.; Weiss, D. E Cin:iurukin, A. (2001) «Atmospheric Pb deposition in Spain during the last 4,600 years recorded by two ombrotrophic peat bogs and implications for the use of peat as a geochemical archiven, The Science of the Total Environment, (en prensa).

Martingz Cortizas, A.; LoOIJARD, A.; Franco Masid:, S. I Garcia-Rodeja, E. (1999)-Complex soil evolution and trace metals: the case of $\mathrm{Hg}$ in the atlantic rankern, in WENZFI., W. W. ADRIANO, D.C. AllowAY, B.; DONER, H.E.; KEJI.ER, C.; LEPp, N.W.; Mench, M.; Naidu, R. Pierzynski, G.M. (eds.), Proc. $5^{t h}$ International Conference on the Biogeochemistry of Trace Elements, Vienna, vol l, p. 106-107.

martinez. Cortizns, A.; Nóvon Muñoz, J.C.; Pontlivedra POMBAL, X.; GarCi A-RODEja, E. E Llana RoDRiguez, C. (1997) - «Paleocontaminación: evidencias de contaninación atmosférica antrópica en Galicia durante los últimos 4.000 años», Gallaecia, 16, ․ 7-22.

Martinez Cortizas, A.; Nóvoa Muñoz, J.C; Pontevedra Pombal, X. E. Garcia-Rodeja, E. (2000) - «Las lurberas como archivos de los procesos crosivos holocenos en Galicia (NW de la Peninsula lbérica)», in DiAz DEL OLOMO, F.; FAUST, D. ı PORRAS, A.I. (eds.), Environmental Changes During the Holocene, Commission on the Holocene, Universidad de Sevilla, Katholische Universität Eichstätt, AEQA, Sevilla, p. 159-162.
Martinez Cortizas, A.; Pérez Alberti, A.; Franco Maside, S. E GArCiA-RodeJA, E. (2000) - «Evolución del paisaje durante el Holoceno en Galicia (NW de la Península Ibérica)y, in DÍAZ DEL. Olomo, F.; Faust, D. E PORRAS, A.I. (eds.), Environmental Changes During the Holocene, Commission on the Holocene, Universidad de Sevilla, Katholische Universität Eichstätt, AEQA, Sevilla, p. 61-64.

Mar'tinez Cortizas, A.; Pontevedra Pombal, X.; Nóvoa Muñoz, J.C. E GARCIA-RODEJA, E. (1997) - «Four thousand years of atmospheric $\mathrm{Pb}, \mathrm{Cd}$ adn $\mathrm{Zn}$ deposition recorded by the ombrotrophic peat bog of Penido Vello (Northwestern Spain)», Water; Air and Soil Pollution, 10, p. 387-403.

Martinez Cortizas, A.; Pontevedra Pombal. X.; Nóvoa Muñoz, J.C.; GARCIA-RoDisa, E. E SHOTYK, W. (1999) - «Mercury in a Spanish peat bog: archive of climate change and atmospheric metal deposition", Science, 284, p. 939-942.

Martinez Cortizas, A.; Ramil Rego, P. e Llana Rodriguez, C. (1993) - «Edafología y Palinologia: apliación al estudio de yacimientos al aire libre en Galicia», Trabalhos de Antropologia e Etnologia, 33, p. 449-469.

Martinez Cortizas, A.; Ramil. Rego, P.; Garcia-Rodija, E. MonRIs Domínguiz, C. (1993) - «Suelos de montaña y ciclos de etabilidad/inestabilidad de las vertientes en Galician, in Pérzz Alibertia., Gutidin Rivera, L., E RAmit. Rego, P. (eds) La evolución del Paisaje en las Montañas del Entorno de los Caminos Jacobeos, Xunta de Galicia, Santiago, p. 107-123.

martinez Cortizas, A.; Ropriguez Guitińn, M.; Ramil Rego, P.; Moares Dominguez, C. (1993) - « Evolución morfogenética y edáfica durante el Tardiglaciar y el Holoceno en las Sierras Septentrionales de Galician, Actas da 3" Reuniao do Quaternário lbérico, Universidad de Coimbra, GTPEQ, AEQUA INQUA, Coimbra, p. 63-71.

monres dominguiz, C.; Tabonda Rodriguez, T.; Garcia-Rodeja, E. E MARTiNI: CORTIZAS, A. (1996) - «Tendencias de la podsolización durante el Cuaternario reciente en las Sierras Septentrionales de Galiciay, in PÉREz ALBERTI, A. E MARTíNEZz CORTIZAS, A. (eds.), Avances en la Reconstrucción Paleoambioental de las äreas de Montaña Lucenses, Diputación Provincial de Lugo, Lugo, p. 121-136.

Pontivindra Pombal, X. Nóvoa Muñoz, J.C.; Garcia-Rodeja, E. ミ MARTiNEZ CORTIZAS, A. (2000) - «Reconstrucción de la contaminación atmosfërica por metales pesados durante el Holoceno en Galicia (NW de España)m, in Dínz det. Olomo, F.; Faust, D. E PORRAS, A.1. (eds.), Environmental Changes During the Holocene, Commission on the Holocene, Universidad de Sevilla, Katholische Universität Eichstätt, $\mathrm{AEQA}$, Sevilla, p. $175-178$.

Ramit., P. (1993) - « » in Pérez Alberti,A., Guitián Rivera, L Ramil Rego, P. (eds.). La Evolución del Paisaje en las Montañas del Entorno de los Caminos Jacobeos. Conselleria de Relaciones Institucionais e Portavoz do Goberno, Xunta de Galicia. p. $25-60$. 